\title{
Mehrfache Vorfeldbesetzung und Informationsstruktur: Eine Bestandsaufnahme ${ }^{1}$
}

\begin{abstract}
Dieses Papier diskutiert informationsstrukturelle Aspekte der mehrfachen Vorfeldbesetzung im Deutschen. Auf der Grundlage einer größtenteils aus den IDS-Korpora extrahierten Belegsammlung werden Diskursgegebenheit, Fokus- und Topikstatus (vor allem) des Vorfeldmaterials beschrieben und in Bezug zu entsprechenden Aussagen in der Literatur gesetzt. Neben informationsstrukturellen Faktoren werden im letzten Abschnitt mögliche weitere Faktoren angesprochen, die mehrfache Vorfeldbesetzung favorisieren könnten. Zudem werden für einen begrenzten Ausschnitt des Deutschen erstmals Zahlen vorgelegt, die das Verhältnis von mehrfacher Vorfeldbesetzung zur ähnlichen, aber als „kanonischer“ geltenden Besetzung des Vorfelds mit einer (möglicherweise partiellen) Verbalphrase illustrieren.

The present paper is a survey of the information-structural properties of multiple fronting constructions in German. Based on a collection of naturally occurring examples (for the most part extracted from the corpora hosted at the Institut für Deutsche Sprache in Mannheim), the prefield material is characterised with respect to givenness, topic and focus status, and the findings are discussed in the light of various proposals from the literature. The final section suggests that a number of other factors, not (or only indirectly) related to information structure, probably play a role in accounting for the phenomenon. In addition, quantitative evidence is presented which illustrates the relation between multiple fronting and VP-fronting for a number of selected structures.
\end{abstract}

\section{Mehrfache Vorfeldbesetzung}

In der theoretischen Literatur wurden verschiedene Arten von Daten diskutiert, die mit Blick auf die angenommene V2-Eigenschaft des Deutschen einer Erklärung bedürfen, darunter Sätze mit vorangestellten Fokuspartikeln wie in (1a) (diskutiert in Jacobs 1986; Büring/Hartmann 2001; Reis 2005; St. Müller 2005b, u.a.) oder Konnektoren vor oder nach der eigentlichen Erstposition wie in (1b) (Pasch et al. 2003; St. Müller 2005b; Breindl 2008, u.a.); vgl. auch van de Velde (1978).

(1a) [Vermutlich] [er] empfiehlt ihr das Buch. (Jacobs 1986, S. 112)

(1b) [Der Bürgermeister] [jedoch] hatte scharfe Augen. (nach Breindl 2008, S. 28)

Eine weitere Klasse von Daten wurde als ,mehrfache Vorfeldbesetzung“ (MVB) diskutiert:

(2a) [Die Kinder] [nach Stuttgart] sollst du bringen. (Engel 1970, S. 81)

(2b) [Zum zweiten Mal] [die Weltmeisterschaft] errang Clark 1965 [...] (Beneš 1971, S. 165, nach Müller 2003, S. 6)

(2c) $[\mathrm{Ihm}][$ den Stern] hat Irene gezeigt. (Eisenberg 2006, S. 399)

1 Diese Arbeit entstand als Teil des DFG-Projekts MU 2822/1-1 (Theorie und Implementation einer Analyse der Informationsstruktur im Deutschen unter besonderer Berücksichtigung der linken Satzperipherie) sowie von Projekt A6 des Sonderforschungsbereich 632 „Informationsstruktur“. Die Identifizierung der in Abshnit 3.2.1 und 3.2.2 besprochenen dominanten Muster erfolgte in Zusammenarbeit mit Philippa Cook und ist in Bildhauer/Cook (2010) ausführlicher beschrieben. Ich danke außerdem Jakob Maché, Stefan Müller, Roland Schäfer und Arne Zeschel für Diskussionen, Kommentare zu diesem Papier und Beiträge zur Datensammlung. 
Der Status von Sätzen wie in (2) ist in der Literatur kontrovers diskutiert worden. Oft sind die Daten, auf denen die Diskussion basiert, konstruiert und werden ohne einen Kontext präsentiert, der Grammatikalitätsurteile möglicherweise verbessern würde (Beispiele und Bewertungen in (3a) aus G. Müller (1998, S. 226); in (3b) und (3c) aus ebd. (2004, S. 218)).

(3a) (ich glaube) Kindern Bonbons gibt man besser nicht

(3b) ?? [Kindern] [Bonbons] sollte man nicht geben.

(3c) * [Dieses billige Geschenk] [der Frau] sollte man nicht geben.

Dass mehrfache Vorfeldbesetzung (MVB) mit einer Vielzahl verschiedener Kategorien und grammatischer Funktionen im Vorfeld möglich ist, zeigt die in St. Müller (2003) präsentierte Belegsammlung. ${ }^{2}$ Unklar sind dagegen weiterhin die Lizensierungsbedingungen dieser Konstruktion. Engel (1970, S. 81), St. Müller (2003, S. 27f.) u.a. weisen auf eine mögliche Rolle informationsstruktureller Faktoren hin. Um dies zu untersuchen, müssen Sätze mit mehrfacher Vorfeldbesetzung im Kontext betrachtet werden, und zu diesem Zweck wurde eine neue Datengrundlage geschaffen, in die Belege zusammen mit vorangehendem und nachfolgenden Sätzen aufgenommen wurden.

Der folgende Abschnitt geht auf die Zusammenstellung dieser Datensammlung ein. Abschnitt 3 bespricht ausgewählte Belege mit Blick auf die Informationsstruktur und bietet damit eine erste Bestandsaufnahme. Abschnitt 4 diskutiert mögliche weitere Faktoren, die mehrfache Vorfeldbesetzung begünstigen. Hier wird auch über ausgewählte Bereiche berichtet, in denen Daten zur mehrfachen Vorfeldbesetzung systematisch erhoben und in Bezug zur einfachen Voranstellungen und VP-Voranstellungen gesetzt wurden.

\section{Die Datensammlung}

Allgemein ergibt sich die Schwierigkeit, Daten zur mehrfachen Vorfeldbesetzung in dem Sinne systematisch zu erheben, dass für eine Stichprobe alle Vorkommen erfasst werden. Da das Phänomen (trotz der zahlreichen Belege, die gefunden werden konnten) vermutlich relativ selten ist, wären für eine effiziente Suche sehr große, syntaktisch annotierte Korpora notwendig. Zwar existieren geparste Korpora für das Deutsche, doch ihre Größe unterscheidet sich um etwa das 2.500 -fache von den größten öffentlich verfügbaren, nichtgeparsten Textsammlungen. ${ }^{3}$ Außerdem ist gerade bei einem ,unkanonischen“ Phänomen wie der mehrfachen Vorfeldbesetzung damit zu rechnen, dass durch eine automatische Annotation nicht alle Fälle identifiziert werden (zumal es viele Fälle mit unklarem Status hinsichtlich der mehrfachen Vorfeldbesetzung gibt, siehe unten).

Die große Mehrheit der Belege wurde aus den IDS-Korpora extrahiert (durch Suchanfragen im Hauptarchiv W). ${ }^{4}$ Dabei wurde nach konkretem lexikalischen Material am Satzanfang gesucht, oft auf der Grundlage der bereits attestierten Daten in St. Müller (2003) und

2 Aufbauend auf Hoberg (1997) und Fanselow (1993) schlägt St. Müller eine syntaktische Analyse vor, die das Phänomen als scheinbar mehrfache Vorfeldbesetzung erklärt. Dabei steht ein leeres Verb mit im Vorfeld, von dem die restlichen Vorfeldelemente abhängen, es handelt sich also um eine VP. Vgl. Müller (2005a) für Diskussion und eine Ausarbeitung in HPSG.

3 Das TIGER-Korpus umfasst ca. 900.000 Wörter, TüBa-D/Z hat ca. 790.000 Wörter. Dagegen umfassen die im Hauptarchiv zusammengefassten Korpora des IDS ca. 2,6 Mrd. Wörter (Stand Nov. 2010).

4 In der aufbereiteten Datensammlung wurde das Vorfeldmaterial manuell für informationsstrukturelle Kategorien annotiert. Zugrunde gelegt wurde das Annotationsschema das SFB 632 Informationsstruktur (Götze et al. 2007; siehe auch Krifka 2007), das die drei Ebenen Givenness, Focus und Topic vorsieht. 
lexikalischen Varianten von diesen. Es handelt sich also nicht um eine repräsentative Sammlung, und sie erlaubt keine statistische Auswertung. Zweck der Sammlung ist vielmehr eine Bestandsaufnahme unter informationsstrukturellen Gesichtspunkten, die Grundlage für weitergehende Forschung sein kann. Zwar ist es möglich, mit der verwendeten Methode eingeschränkte Domänen systematisch zu erfassen, indem man sich auf Satzinitiale Sequenzen einer bestimmten Form konzentriert (z.B. ADV+PP oder NP+NP) und dabei nach bestimmten Kriterien lexikalisches Material einsetzt (z.B. semantisch ähnliche Adverbien, verschiedene Determinierer etc.), eventuell in Kombination mit bestimmten Verben (vgl. Abschnitt 4). Bei einer exhaustiven Erfassung entsteht jedoch die Schwierigkeit, für jedes extrahierte Satzexemplar entscheiden zu müssen, ob es sich um einen Fall mehrfacher Vorfeldbesetzung handelt. Dies ist keine triviale Aufgabe, und je nach Annahmen über (un-)mögliche Phrasenstrukturen können Entscheidungen in einigen Fällen unterschiedlich ausfallen, so dass möglicherweise nicht jeder alle Belege für mehrfache Vorfeldbesetzung als solche akzeptieren wird. Ein Beispiel für einen problematischen Fall sind ADV+PP-Sequenzen. Ein syntaktischer Test dafür, ob ein Adverb eine Präpositionalphrase modifizieren kann, ließe sich in Anlehnung an den von Jacobs (1986) vorgeschlagenen Test für die Sequenz PARTIKEL+NP konstruieren. Jacobs argumentiert, dass eine solche Sequenz nur dann als Konstituente (als modifizierte Nominalphrase) zu analysieren ist, wenn sie wie andere Nominalphrasen unter einer Präposition eingebettet werden kann (was Jacobs für die von ihm diskutierten Fälle verneint; vgl. aber St. Müller (2005b) und Bouma/Hendriks/Hoeksema (2007) für entsprechende Belege). Analog dazu lässt sich erwarten, dass die Sequenz ADV+PP innerhalb einer Nominalphrase auftreten kann, wenn es sich bei ihr um eine Konstituente handelt. Die Ergebnisse eines solchen Tests sind jedoch nicht immer eindeutig, wie die Beispiele in (4)-(6) zeigen.

(4a) [Vermutlich] [mit einem Nachschlüssel] drangen unbekannte Täter in ein Wohnhaus in Grödig ein. (I96/OKT.40042)

(4b) ? ihr Eindringen vermutlich mit einem Nachschlüssel

(5a) [Erstaunlicherweise] [aus dem Schulbereich] kommt eine Studie darüber, warum Studenten häufig ihr Studium abbrechen. (K98/JUL.51638)

(5b) ? eine Studie/das Kommen erstaunlicherweise aus dem Schulbereich

(6a) [Anscheinend] [mit einer Rennstrecke] hat ein Autofahrer eine Bahnbaustelle in der Pfalz verwechselt. (M06/OKT.80757)

(6b) ?? eine Verwechslung anscheinend mit einer Rennstrecke

(7a) [Plötzlich] [in den schönen Traum] platzt eine Schlagzeile aus den Fernsehnachrichten. (O97/AUG.88382)

(7b) ?? ihr Hereinplatzen plötzlich in den schönen Traum

Ein weiteres Beispiel für einen Zweifelsfall sind Sätze, in denen das Adverb weiterhin zusammen mit weiterem Material im Vorfeld steht. Pasch et al. (2003, S. 511) listen weiterhin als Konnektor, der die Vorerstposition besetzen kann (so dass entsprechende Fälle nicht als mehrfache Vorfeldbesetzung zählen sollten), allerdings nur zusammen mit einem nicht-Subjekt in der Erstposition. In den Belegen in (8) folgt jedoch auf weiterhin ein

Darüber hinaus sind syntaktische Kategorie und grammatische Funktion der Vorfeldkonstituenten annotiert. Die aus den IDS-Korpora extrahierten Belege sind unter clarin.ids-mannheim.de/SFB632/A6 öffentlich zugänglich und können einschließlich Annotation mit dem ANNIS-Werkzeug (Zeldes et al. 2009) durchsucht werden. 
Subjekt, so dass es sich in diesen Fällen entweder nicht um den Konnektor weiterhin handelt, oder die von ebd. genannte Beschränkung des Konnektors weiterhin dahingehend gelockert werden muss, dass zumindest bestimmte Subjekte in der Erstposition auftreten können.

(8a) [Weiterhin] [alles] [optimal] läuft beim HC Davos. (A01/OKT.35466)

(8b) [Weiterhin] [Ärger] droht dem Coach und den Freezers indes aus Österreich. (HMP06/ NOV.03145)

(8c) [Weiterhin] [eine volle Klasse] wird im neuen Schulhaus Gill im Kreis Kappel unterrichtet. (A99/JUN.41466)

(8d) [Weiterhin] [Misstöne] herrschen zwischen Wien und Mailand. (K00/MAR.18528)

In den folgenden Abschnitten werden Sätze mit mehrfacher Vorfeldbesetzung unter informationsstrukturellen Gesichtspunkten erörtert. Der Status der präsentierten Belege als Instanzen mehrfacher Vorfeldbesetzung wird hier nicht weiter thematisiert (siehe St. Müller (2003), der verschiedene Klassen von Beispielen diesbezüglich diskutiert).

\section{Informationsstruktur in Sätzen mit MVB}

Die Einordnung der Belege in diesem Abschnitt orientiert sich an den in Krifka (2007) vorgeschlagenen informationsstrukturellen Dimensionen Gegebenheit, Fokus und Topik/ Delimitation, sowie an deren Ausarbeitung zu einem Annotationsschema von Götze et al. (2007).

\subsection{Gegebenheit}

Götze et al. (2007) setzen für Gegebenheit die Ausprägungen ,gegeben“, „zugänglich“ und „neu“ an, die ersten beiden davon mit (optionaler) weiterer Untergliederung. Ein Diskursreferent gilt als gegeben, wenn ein vorangehender Ausdruck ebenfalls darauf verweist. Liegt dieser Ausdruck im selben oder im unmittelbar vorangehenden Satz, zählt der Diskursreferent als aktiv, andernfalls als inaktiv. Ein Referent ist zugänglich, wenn er entweder situativ, durch Weltwissen oder durch eine Relation zu anderen vorerwähnten oder zugänglichen Diskursreferenten erschlossen werden kann. ${ }^{5}$ Ist dies nicht der Fall, gilt ein Diskursreferent als neu.

Eine Angabe der Diskursgegebenheit ist nur für referierende Ausdrücke vorgesehen. Für die mehrfache Vorfeldbesetzung finden sich häufig Belege, bei denen eine der Konstituenten im Vorfeld ein Adverb oder ein Bestandteil eines Idioms ist. Für beide ist Gegebenheit nicht definiert, so dass die Darstellung hier nur NPen (einschließlich solcher, die in PPen eingebettetet sind) im Vorfeld berücksichtigt. ${ }^{6}$

5 Hier kommen u.a. Teil-Ganzes- und verschiedene Mengenrelationen in Frage; vgl. Götze et al. (2007, S. 156-160).

6 Bei einigen Idiomen lassen sich jedoch einzelnen Bestandteilen Bedeutungen zuordnen, so dass diese dann metaphorisch referieren können (entsprechend ,,idiomatically combining expressions“ bei Nunberg/Sag/Wasow 1994). Dies scheint auch bei den weiter unten angesprochenen Idiomen Öl ins Feuer gießen und das Fass zum Überlaufen bringen der Fall zu sein. 


\subsubsection{Gegebene Diskursreferenten}

Belege, in denen die Diskursreferenten beider Vorfeldelemente den Status „gegeben“ haben, wurden bis jetzt nicht gefunden. Gelegentlich ist eines der Vorfeldelemente gegeben, während das andere entweder zugänglich, neu oder überhaupt nicht referenziell ist. In Beispiel (9) ist der Diskursreferent des ersten Vorfeldelements aktiv, in (10) dagegen der des zweiten.

(9) Ganz allein können die Rotkreuzler, solch einen Blutspendetag nicht ausführen.

[Ihnen $]_{\mathrm{i}}$ [zur Seite] stehen stets Mitarbeiter des DRK-Blutspendedienst Baden-Württemberg-Hessen. (M05/APR.27791)

(10) Ballack aber wirkte fahrig und gehemmt und vermochte dem Spiel ${ }_{i}$ seinen Stempel nicht aufzudrücken.

[Schwung] [ins Spiel $]_{\mathrm{i}}$ brachte neben Trochowski vor allem der unermüdliche Bastian Schweinsteiger. (http://www.sueddeutsche.de/,tt5m1/sport/319/314219/text, 15/10/2008)

In den Beispielen (11) und (12) ist der Diskursreferent des ersten bzw. des zweiten Vorfeldelements inaktiv. ${ }^{7}$

(11) Beim Apéro der Jubiläumsfeier ${ }_{i}$ vom Sonntag, die vom Entertainer Bruno Höck musikalisch umrahmt wurde, bedankte sich Monika Frei für die Treue der Gäste und die vielen schönen Begegnungen. [...] Speziell erwähnte Monika Frei Käthi Aegerter, die als Servicemitarbeiterin seit Beginn dabei ist. Ausstellung zum Jubiläum

[Dem Jubiläum $]_{\mathrm{i}}$ [eine besondere Note] verliehen die Bilder und Skulpturen der in Lenggenwil aufgewachsenen Künstlerin Maria Fallo [...] (RHZ05/JUN.03263)

(12) Doch sollte jeder Mensch mit einem Blutdruck über $130 \mathrm{zu} 85$ mit entsprechender Ernährung versuchen, die Werte niedrig zu halten. An erster Stelle sollten Übergewicht abgebaut und der Kochsalzverbrauch eingeschränkt werden.

[Ungünstig] [auf den Blutdruck] $]_{i}$ wirke sich auch regelmäßiger Alkoholkonsum aus. (M02/ MAR.22187)

\subsubsection{Zugängliche Diskursreferenten}

Am Übergang von ,gegeben“ zu ,zugänglich“ liegt der Status des Diskursreferenten von „Saft" in (13).

(13) Unverzichtbar aber sind Pomeranzen (5,50 pro Kilo) für Orangenmarmelade. Bloß: Wer kocht die schon noch zu Hause selbst? Wenig Mühe macht dagegen das Saftpressen, und so werden die dafür speziell gezüchteten Früchte, wie spanische Salusianas, gern gekauft.

[Dem Saft] [eine kräftigere Farbe] geben Blutorangen, die zur Zeit aus Sizilien stammen. (R99/JAN.01605)

In (14) ist der Diskursreferent des ersten Vorfeldelements nicht gegeben, er lässt sich jedoch als Aggregation der individuellen Diskursreferenten verstehen, die durch die vier vorangehenden Propositionen eingeführt wurden (angedeutet in geschweiften Klammern), und ist somit zugänglich.

(14) \{Kaffeeduft wird durch die Linden strömen, $\}_{1}$ und \{bei der Musik von Johann Strauß, bei leckerem Kuchen und einer Tasse Kaffee kann man es sich gut gehen lassen. $\}_{2}$ \{Der Klas-

7 Eine Frage, die sich für Zeitungstexte bei der Annotation von Gegebenheit stellt, betrifft den Status von Titeln und Zwischenüberschriften. Zählt man die Zwischenüberschrift in (11) mit, dann ist der Diskursreferent von „Jubiläum“ im nachfolgenden Satz als aktiv einzuordnen. Vgl. auch die Beispiele (15) und (18) unten. 
siker im Programm des Kulturfördervereins erfreut sich größter Beliebtheit $\}_{3}$ und \{wird musikalisch in diesem Jahr am 15. Juli vom Frankfurter Johann-Strauß-Ensemble gestaltet. $\}_{4}$

$[\text { Dem Ganzen }]_{1+2+3+4}$ [ein Sahnehäubchen] setzt der Solist Klaus Durstewitz auf [...] (NON08/ FEB.08467)

In (15) ist es plausibel, den Referenten des zweiten Vorfeldelements als zugänglich zu analysieren (erschließbar als Teil einer Kommunikationssituation im April). Das erste Vorfeldelement wäre hier als neu einzustufen (es sei denn, das Vorkommen von „LiveMusik“ im Titel wird mit einbezogen; in diesem Fall würde das erste Vorfeldelement als aktiv zählen).

(15) Live-Musik im „Wexel“ Edingen

[Mit Live-Musik] [in den Mai] geht es am Freitag, 30. April, im Bistro „Wexel“ in Edingen [...] (M04/APR.28800)

Für Beispiel (16) ist es plausibel anzunehmen, dass die Diskursreferenten beider Vorfeldelemente zugänglich sind (Weltwissen über Fußballspiele macht die Referenten von „Führung“ und „Kabine“ eindeutig identifizierbar).

(16) Lange Zeit schien sich im Spitzenduell und Nachbarderby der 2. Klasse Süd zwischen Eben und Forstau eine alte Fußball-Weisheit zu bewahrheiten: „Tore, die man nicht schießt, die bekommt man." Die Gäste aus Forstau, Tabellenführer der 2. Klasse Süd, hatten nämlich auf dem generalsanierten Platz von Verfolger Eben 45 Minuten lang Chancen am laufenden Band vernebelt - dazu ihr Stürmer Hannes Helpferer nur ans Torholz geköpfelt (25.).

[Mit der Führung] [in die Kabine] war jedoch Eben gegangen (N00/OKT.45268)

Die Beispiele in (9)-(15) haben die Gemeinsamkeit, dass eines der Vorfeldelemente etwas Gegebenes oder Zugängliches wieder aufnimmt, während das andere Vorfeldelement im weitesten Sinn einen neuen Aspekt, oft einen Kontrast, in Bezug darauf einbringt. Diese Konfiguration scheint sich bei mehrfachen Vorfeldbesetzungen häufig zu finden, sie ist jedoch nicht zwingend, wie die Daten im folgenden Abschnitt zeigen.

\subsubsection{Neue Diskursreferenten}

Während die Daten in (10)-(11) und (13)-(15) zeigen, dass Mehrfachbesetzungen möglich sind, bei denen ein Referent neu eingeführt wird, sind in den Beispielen (17) und (18) die Diskursreferenten beider Vorfeldelemente neu. ${ }^{8}$

(17) Das Naturschutzgebiet Backofen-Riedwiesen zwischen Mannheim und Brühl birgt Kostbarkeiten der Fauna und Flora, die man in der Nähe einer Großstadt nicht vermutet. Ein unerfahrener Spaziergänger erblickt sie, wenn, dann nur durch einen Zufall. Mit einem Naturschutzbeauftragten unterwegs, erschließt sich die Vielfalt. Das erfahren die SafariTeilnehmer am 7. August.

[Mit einem Fahrrad] [auf ausgewiesenen Wegen] geht's hinein in das Auengebiet (M01/ JUL.51031)

(18) „Billard“ mit Autos

[Mit seinem Lkw] [auf eine stehende Fahrzeugkolonne] fuhr Paul S. (51) aus Absam auf. (O97/NOV.94276)

\footnotetext{
8 Wie in (15) oben hängt die Klassifizierung von (18) davon ab, ob Ausdrücke in Zwischenüberschriften als Antezedens gelten. „Autos“ könnte dann den Referenten von „Fahrzeugkolonne“ zugänglich machen. Stefan Müller hat mich darauf hingewiesen, dass es sich beim Vorfeld in (17) möglicherweise um Koordination handelt, es liegt hier also nicht eindeutig mehrfache Vorfeldbesetzung vor.
} 


\section{Felix Bildhauer}

\subsubsection{Gegebenheit und Voranstellung von Idiomteilen}

Häufig ist das zweite Vorfeldelement Bestandteil eines Idioms. Das erste Element kann ebenfalls Teil des Idioms sein, wie in (22), oder es kann sich um anderweitig referenzielles oder nicht-referenzielles Material handeln. Nicht-idiomatische referenzielle Konstituenten, die zusammen mit einem nicht-referenziellen Bestandteil eines Idioms vorangestellt sind, können sich auf gegebene, zugängliche und neue Diskursreferenten beziehen, wie die Beispiele in (19)-(21) zeigen. In (22) ist dagegen keine der Konstituenten im Vorfeld referenziell.

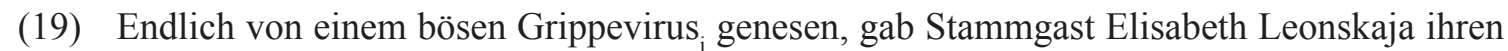
Klavierabend in Deutschlandsberg.

[Dem Virus], [zum Opfer] fiel leider das mit Spannung erwartete Mittelstück, Prokofjews 6. Sonate. (K99/MAI.30694)

(20) [...] Neuberger Ortsteil Ravolzhausen: Polizeiangaben zufolge war dort kurz nach 15 Uhr aus bisher unbekannter Ursache in einer Doppelhaushälfte ein Feuer ausgebrochen, das sich schnell auf das ganze Haus ausbreitete.

[Den Flammen] [zum Opfer] fielen eine komplette Wohnung sowie der gesamte Dachstuhl. (N97/SEP.35831)

(21) Brand im Schulabfall Schriesheim

[Einer Brandstiftung] [zum Opfer] fielen in der Nacht zum Dienstag Papierabfallbehälter im Außenbereich der Kurpfalz-Realschule. (M00/MAI.22963)

(22) [Die Flinte] [ins Korn] will der Vorarlberger wegen des verpatzten Auftritts nicht werfen. (K00/SEP.69749)

Zusammenfassend lässt sich sagen, dass die beiden Vorfeldelemente, sofern sie referieren, sich auf gegebene, zugängliche und neue Diskursreferenten beziehen können. Dabei sind fast alle Kombinationen belegt, mit Ausnahme derjenigen mit zwei gegebenen Referenten. Häufig ist die lineare Abfolge dabei: given $<$ accessible $<$ new; jedoch finden sich auch andere Fälle, vgl. (10) und (15).

\subsection{Fokus}

Mit Rooth $(1985,1992)$ und Krifka (2007) soll hier angenommen werden, dass die Funktion von Fokus darin besteht, Alternativen einzuführen. Krifka hebt hervor, dass diese Definition sowohl semantischen Fokus (mit Einfluss auf die Wahrheitsbedingungen einer Äußerung) als auch pragmatischen Fokus (diskursstrukturierend, ohne Einfluss auf die Wahrheitsbedingungen) abdeckt.

Unter den MVB-Belegen finden sich einige Fälle, in denen ausschließlich das Vorfeldmaterial Träger der Fokusmarkierung ist. So ist es für Beispiel (23b) plausibel anzunehmen, dass der Hauptakzent auf gleichzeitig fallen würde, während das (diskurs-gegebene) Subjekt im Mittelfeld deakzentuiert ist (die a- und c-Zeilen liefern den Vor- und Nachkontext).

(23a) Was für Hoffnungen hat Ehud Barak ausgelöst, als Israel endlich wieder einen Premier hatte, der den Frieden wagen wollte.

(23b) [Alle Fronten] [gleichzeitig] wollte der Ex-Generalstabschef begradigen, Abkommen mit Syrien, Libanon und den Palästinensern schließen.

(23c) Ein Jahr später steht Barak vor einem Scherbenhaufen: [...] (P00/AUG.28493) 
Der Fokus in (23b) kann als pragmatischer Fokus mit diskursstrukturierender Funktion analysiert werden, da er nicht mit einem Operator assoziiert wird und sich dementsprechend auch nicht auf die Wahrheitsbedingungen auswirkt. Es stellt sich die Frage, ob und wie weit der Fokus in solchen Fällen projiziert, d.h. ob Material, das über den prominenten Fokusexponenten hinausgeht, im jeweiligen Kontext ebenfalls als fokussiert betrachtet werden kann. Bei (23b) muss man davon ausgehen, dass die gesamte VP fokussiert ist, denn der Satz beantwortet (implizite) Fragen wie „Was hat Barak getan?“" (vgl. Bemerkungen zu Fokusprojektion und Akkommodierung von Diskursfragen unten). Demnach liegt hier Fokusprojektion vor. Beispiel (23b) kann als Fall von pars-pro-toto-Voranstellung (Fanselow 2004) analysiert werden, bei welcher der Akzentträger einer fokussierten Konstituente stellvertretend für die gesamte fokussierte Konstituente im Vorfeld steht.

Belege wie (23b) scheinen jedoch untypisch für mehrfache Vorfeldbesetzung zu sein. Stattdessen wird die Belegsammlung von zwei Mustern dominiert (vgl. Bildhauer/Cook 2010), die in (24b)-(25b) und (26b)-(27b) illustriert sind. In den jeweiligen Kontexten würde in diesen Sätzen der Hauptakzent wohl auf ein Element im Mittelfeld fallen. In (24b) und (25b) handelt es sich um das Subjekt, in (26b) und (27b) um ein Adverbial.

(24a) Der FC Augsburg ist auch im DFB-Pokal nicht zu stoppen und steht erstmals in seiner Geschichte in der Runde der besten Vier. Die Schwaben setzten sich am Mittwoch in einer knüppelhart geführten Partie mit 2:0 (1:0) gegen den 1. FC Köln durch. [...] Nach dem Seitenwechsel waren nur wenige Sekunden gespielt, als Thurk mit einer Kopie seines 1:0 beinahe für die Vorentscheidung gesorgt hätte. Wieder flankte Brinkmann von rechts, wieder kam der Stürmer aus kurzer Distanz mit dem linken Fuß an den Ball - doch diesmal klärte Keeper Faryd Mondragon mit einem überragenden Reflex.

(24b) [Den Kölnern] [einen Bärendienst] erwies nach etwas mehr als einer Stunde ausgerechnet Nationalspieler Podolski,

(24c) der wegen einer Fußblessur zunächst auf der Bank Platz nehmen musste. Kaum neun Minuten auf dem Feld, provozierte der Stürmer-Star nach einem Foul Schiedsrichter Kinhöfer derart [...] (http://www.haz.de/Nachrichten/Sport/Fussball/Uebersicht/FC-Augsburggelingt-Coup-gegen-acht-Koelner, 10/02/2010)

(25a) Um auf Nachfrageschwankungen reagieren zu können, hat Siemens ein modulares Investitionsprogramm aufgelegt: langfristig geplante Großvorhaben, die je nach Marktlage durch kurzfristige Ausbaumaßnahmen ergänzt werden.

(25b) [Stabilisierend] [auf das Unternehmen] wirkte in den vergangenen Jahren laut Hinkel das Ausland.

(25c) „Denn da ist die Dose noch immer hoch angesehen.“ (RHZ09/JUL.07639)

(26a) Der Klosterpalast von Mafra verfügt über 1200 Räume und verschlang ein Viertel des Goldes, das Portugal aus seiner damaligen Kolonie Brasilien herauszog. Dieser Prunk mag zwar Kreml-Chef Wladimir Putin beeindrucken.

(26b) [Positiv] [auf die Stimmung] dürfte er sich kaum auswirken.

(26c) Das Verhältnis sei „so schlecht wie seit dem Kalten Krieg nicht mehr“, heißt es in Brüssel. (RHZ07/OKT.24521)

(27a) Um diese Zeit zu erreichen, muss sie auch ihre persönliche Bestleistung über $400 \mathrm{~m}, 59,41$ Sekunden, verbessern.

(27b) [Ganz] [auf $400 \mathrm{~m}$ ] wird sie sich aber nie konzentrieren,

(27c) da sie wegen ihrer Grundschnelligkeit schneller an ihre Grenze stösst als über 800 m. (A98/ JUN.38727) 


\subsubsection{Subjekt im Fokus}

Wie (23b) können auch (24b) und (25b) als Sätze analysiert werden, in denen der Fokus nicht mit einem Operator assoziiert ist, sich nicht auf die Wahrheitsbedingungen auswirkt und deshalb nur pragmatische (diskursstrukturierende) Funktion hat. Im Fall von (24b) erfordert der Diskurs an dieser Stelle eine Antwort auf Fragen wie „Was passierte dann?“, „Was war mit den Augsburgern?“ oder „Was war mit den Kölnern?“. Legt man diese Fragen zugrunde, müsste der Fokus in (24b) mindestens das Subjekt, das Verb und das direkte Objekt einschließen (den Kölnern kann man als Hintergrund auffassen), demnach läge also Fokusprojektion ausgehend vom Subjekt vor. ${ }^{9}$ Eine andere Möglichkeit besteht darin, anzunehmen, dass der Fokus in (24b) nicht projiziert und der Satz stattdessen die speziellere (implizite) Frage „Wer erwies den Kölnern einen Bärendienst?“ beantwortet. Da sich eine solche Frage nicht ohne weiteres aus dem Vorkontext ergibt, muss man dann davon ausgehen, dass sie akkommodiert wird; bei dieser Erklärung stellt (24b) eine neue Frage zur Diskussion und gibt gleichzeitig die Antwort darauf. ${ }^{10}$

Das Muster tritt jedoch auch diskursinitial auf, wie in (28b). Äußerungen, bei denen der Sprecher keine spezifischen Annahmen über den Inhalt des Common Ground macht (,outof-the-blue“-Äußerungen, ,,all-new“"- oder „,news“-Sätze), werden in der Literatur häufig als Strukturen mit weitem Fokus (Satzfokus) behandelt (Selkirk 1995; Krifka 2007, u.v.a.). Zwar könnte man auch für (28) argumentieren, dass keine Fokusprojektion vorliegt, so dass statt der Frage „Was ist passiert?“ die Frage,,Wer stürzte gestern mit seinem Paragleitschirm in den Tod?" beantwortet (und akkommodiert) wird. Mit einem solchen Schritt gibt man jedoch ein Diagnosekriterium für Satzfokus auf, nämlich die Möglichkeit, dass ein Satz als Antwort auf sehr allgemeine Fragen wie „Was ist passiert?“ oder „Was gibt‘s neues?" dienen kann.

(28a) Paragleiter stürzte in den Tod

(28b) [Mit dem Paragleitschirm] [in den Tod] stürzte gestern ein 43jähriger Deutscher.

(28c) Er war am Nachmittag beim „Steinerhof“ gestartet. (I97/MAI.17740)

$9 \quad$ Man könnte auch das direkte Objekt im Vorfeld als weitere fokussierte Konstituente auffassen, da es wohl auch einen Akzent tragen müsste (eine natürliche Realisierung des Satzes scheint die Hutkontur zu sein). Unter dieser Annahme könnte auch das direkte Objekt Ausgangspunkt der Fokusprojektion sein, und (24b) ließe sich ähnlich wie (23b) als Fall von pars-pro-toto-Voranstellung beschreiben, jedoch mit zwei Unterschieden: Erstens ist in (24b) nicht die gesamte VP fokussiert, stattdessen steht genau der Teil der VP, der nicht fokussiert ist (die Dativ-NP), ebenfalls mit im Vorfeld. Zweitens ist hier, im Gegensatz zu (23b), das Subjekt ebenfalls Teil des Fokus. Möglicherweise ist dieser Mismatch zwischen informationsstrukturellen und syntaktischen Partitionen einer der Faktoren, die mehrfache Vorfeldbesetzung in solchen Fällen motivieren.

10 Krifka (2007) verwendet „,accommodation“ für alle impliziten, diskurssteuernden Fragen, die durch Foki angezeigt werden, nicht nur für „unerwartete“. Daneben können bei ihm auch einzelne Propositionen akkommodiert und in den Common Ground aufgenommen werden, vorausgesetzt es handelt sich dabei um unkontroverse Tatsachen. Analysiert man das Subjekt in (24b) als eng fokussiert und versteht den Satz als Antwort auf die implizite Frage „Wer hat den Kölnern einen Bärendienst erwiesen?“, dann muss man annehmen, dass für die Akkomodierung von Propositionen und diskursstrukturierenden Fragen nicht die gleichen Voraussetzungen gelten: Die für (24b) zu akkommodierende Frage muss im gegebenen Diskurs als unerwartet gelten und präsupponiert Information (jemand hat den Kölnern einen Bärendienst erweisen), die wohl mindestens ebenso relevant für den Fortgang des Diskurses ist, wie der tatsächlich assertierte Teil des Satzes (jemand = Lukas Podolski). 
Sätze mit mehrfacher Vorfeldbesetzung, bei denen das Subjekt im Mittelfeld fokussiert ist (eng oder als Teil eines größeren Fokus), treten sehr häufig mit neuen Subjekten auf, deren Referenten auf diese Weise in den Diskurs eingeführt werden und (typischerweise) als Topik in nachfolgenden Sätzen fungieren (vgl. Bildhauer/Cook (2010) für eine detailliertere Diskussion dieses Musters und eine formale Analyse). Wenn der Satz diskursinitial ist, folgen dem Subjekt im Mittelfeld oft noch weitere Konstituenten, die wohl auch einen Akzent bekommen müssten, so dass in diesen Fällen der letzte Akzent/Hauptakzent nicht mehr auf das Subjekt fallen würde und demnach keine Fokusprojektion ausgehend vom Subjekt vorliegt, wie in den Beispielen (29b) und (30b). Da die diskursstrukturierende Funktion von solchen Sätzen (typischerweise Einführung eines neuen Subjekt-Referenten und nachfolgende Wiederaufnahme als Topik) identisch zu sein scheint mit Sätzen wie (28), bietet es sich an, solche Fälle von mehrfacher Vorfeldbesetzung in einer Kategorie zusammenzufassen. Die formale Gemeinsamkeit besteht dann darin, dass ein Subjekt im Mittelfeld innerhalb des Fokusbereichs liegt.

(29a) Feldbach

(29b) [Mit beiden Händen] [in die Kreissäge] geriet Mittwoch nachmittag Karl L. aus Gnas beim Holzarbeiten in seiner Firma.

(29c) Der 32jährige erlitt schwerste Schnittverletzungen. (O94/MAR.24110)

(30a) Treppensturz

(30b) [Mit dem Gesicht] [auf eine Steinplatte] schlug Mittwoch nachmittag Simone P. (24) aus Tösens in ihrem Garten.

(30c) Sie wurde dabei schwer verletzt. (O96/MAI.55771)

\subsubsection{Adverbial im Fokus}

Bei den Beispielen (26b) und (27b) ist ein Adverb im Mittelfeld fokussiert, während das Subjekt im Mittelfeld in beiden Fällen diskursgegeben ist. Bei dem Adverbial handelt es sich sehr häufig um die Negation nicht, die beiden Beispiele zeigen jedoch, dass auch andere Adverbien möglich sind (vgl. Bildhauer/Cook 2010). Typischerweise ist eines der beiden Elemente im Vorfeld kontrastiv, es gibt hier also noch einen zweiten Fokus im Satz; die Struktur lässt eine Lesart mit Skopusinversion zu, eine typische Eigenschaft von $i$-Topikalisierungen (Jacobs 1997; Krifka 1995), wie Beispiel (31) zeigt (allerdings mit Subjekt im Vorfeld).

(31) Die Kinder haben eigene Familien gegründet und wohnen alle einigermaßen in der Nähe, so daß die Jubilarin ihre 19 Enkel- und 17 Urenkelkinder häufig sehen kann.

„,[Alle] [gleichzeitig] können mich nicht besuchen, weil ich gar nicht so viel Platz habe“, lacht sie. (V99/JAN.02701)

Es ist für diese Sorte mehrfacher Vorfeldbesetzung nicht ganz einfach, den Fokusbereich genau abzugrenzen. Welche (implizite) Frage zeigen die Sätze an? Eine Möglichkeit besteht darin, in diesen Fällen das Adverbiale als eng fokussiert zu betrachten. In den Beispielen (26b) und (27b) wären dann die impliziten Fragen:

(26') Inwieweit trifft es zu, dass der Prunk sich positiv auf die Stimmung auswirkt?

(27') Inwieweit trifft es zu, dass sie sich einmal ganz auf 400m konzentrieren wird? 
Man müsste dann allerdings davon ausgehen, dass die Fragen akkommodiert werden, zumal sie im jeweiligen Kontext als unerwartet gelten können. Eine andere Möglichkeit ist, für solche Beispiele Fokus auf Teilen der VP anzunehmen. Das Subjekt im Mittelfeld kann als Teil des Hintergrunds angesehen werden, und zumindest im Fall von (27b) kann auch das vorangestellte direkte Objekt als Teil des Hintergrunds gelten. Unter dieser Analyse würde die Fokusstruktur anzeigen, dass Fragen wie die folgenden zur Diskussion stehen:

(26”) Was gibt es noch über den Prunk (in Bezug auf die Stimmung) zu sagen?

(27") Was gibt es noch über sie in Bezug auf die 400m zu sagen?

Im Gegensatz zu (24b) und (25b) dienen Sätze wie (26b) und (27b) nicht dazu, einen neuen Diskursreferenten einzuführen und damit einen Topikwechsel vorzubereiten; aus dem Nachkontext der Sätze ist ersichtlich, dass sich das Topik nicht ändert. Im Zusammenhang mit einer anderen Voranstellungskonstruktion (die gemeinsame Voranstellung eines Subjekts und eines nicht-finiten Verbs) schlägt Cook (2010) eine Analyse als invertierte TopikKommentar-Struktur vor, bei der bis auf ein Adverb (eines bestimmten Typs) und ein topikales Element im Mittelfeld das gesamte Material dem Kommentar zugerechnet wird. Das Adverb dient als Bindeglied zwischen Topik und Kommentar und quantifiziert, in welchem Maße der Kommentar auf das Topik zutrifft. Bildhauer/Cook (2010) übernehmen diese Analyse in ihre formale Beschreibung der mehrfachen Vorfeldbesetzung.

Die Beispiele in diesem Abschnitt illustrieren, dass zumindest eines der Vorfeldelemente typischerweise fokussiert oder Teil eines größeren, möglicherweise diskontinuierlichen Fokus ist. Die genaue Bestimmung des Fokusbereichs ist dabei in einigen Fällen nicht eindeutig und hängt von Annahmen über die Rolle von impliziten diskursstrukturierenden Fragen und deren Akkommodierbarkeit ab. Charakteristischer als die Rolle der vorangestellten Elemente in Bezug auf Fokus ist bei der mehrfachen Vorfeldbesetzung die Fokussierung von Elementen im Mittelfeld, bei der die zwei oben skizzierten Muster zusammengenommen einen Großteil der Daten abdecken. In einigen Fällen (so z.B. in (24b)) ist mehrfache Vorfeldbesetzung möglicherweise dadurch motiviert, einen Fokusbereich über adjazentes Material herzustellen, zumal in geschriebener Sprache derartige Markierungen nicht prosodisch realisierbar sind (vgl. St. Müller (2005b) zur disambiguierenden Positionierung von Partikeln in geschriebener Sprache).

\section{$3.3 \quad$ Topik}

Legt man als Definition von Topik die Adressierungsfunktion zugrunde (Jacobs 2001), und geht man davon aus, dass nur referierende Ausdrücke diese Funktion haben können, dann geht aus den bislang diskutierten Daten hervor, dass nicht beide Vorfeldelemente Topiks sein müssen. Derartige Fälle scheinen sogar ausgesprochen selten zu sein; einer der raren Belege ist in (32) wiedergegeben. Für beide Vorfeldelemente lässt sich hier argumentieren, dass der restliche Satz als Prädikation ,über“" sie verstanden werden kann.

(32) Das Darmstädter Jazzinstitut verfügt im Kavaliershaus über einen schönen Gewölbekeller ${ }_{i}$, der geradezu ideal für kleine, feine Konzerte ist: Nicht so klein, daß man sich gleich auf die Füße tritt, wenn mal jemand Bekannteres kommt, aber auch nicht so groß, daß es störendes Echo gäbe. Institutsleiter Wolfram Knauer hat dem Keller ${ }_{i}$ eine Gesprächskonzert-Reihe und die Reihe Jazz-Profile ins Gewölbe geschneidert. Äußerst angemessen ist das Quartett

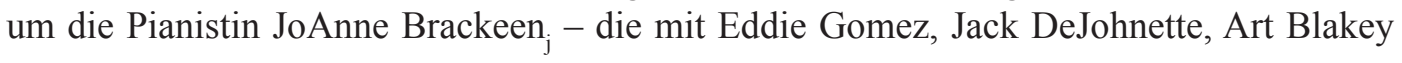


und Joe Henderson gespielt hat - und den Saxophonisten Greg Osby ${ }_{k}$, der seit den achtziger Jahren zu den sogenannten young lions der amerikanischen Ostküste gezählt wurde, zu den Schlüsselfiguren der M-Base gehörte und somit eher zum traditionalistischen Flügel der great black music. Dazu paßt, daß er sich heute verstärkt mit Duke Ellington, Charlie Parker, Thelonious Monk und Sonny Rollins befaßt.

$[\text { Mit den beiden }]_{j+k}[\text { in den Keller }]_{\mathrm{i}}$ kommen Scott Colley am Baß und Horacio Hernandez, Schlagzeug. (R99/MAI.41177)

Dagegen finden sich zahlreiche Fälle, in denen eines der Vorfeldelemente ein AboutnessTopik ist; Beispiele hierfür sind u.a. (9)-(14), (24b) und (25b). Speyer (2008) beobachtet anhand von Daten aus dem Frühneuhochdeutschen, dass Topiks im doppelten Vorfeld stets als zweites Element auftreten, d.h. nach rahmensetzenden und kontrastiven Elementen, und er legt nahe, dass dies auch den Verhältnissen im heutigen Deutsch entspricht. Dabei kann es sich jedoch allenfalls um eine Tendenz handeln, denn Daten wie in (11), (13) und (33) zeigen, dass bei entsprechendem Kontext auch die umgekehrte Abfolge möglich ist.

(33) Im Schlussabschnitt war den Berlinern das Bemühen durchaus anzumerken, vor ausverkauftem Haus ein Debakel zu verhindern.

[Dem Spiel] [eine Wende] konnten sie aber nicht mehr geben. (NUZ07/MAI.01360)

(34) Stadtammann Josef Hartmann dementiert Gerüchte, am Spital Wil sei eine zweite Person mit dem Aids-Virus angesteckt worden: „Es liegt keine Infektion vor.“ Die angeblich betroffene Person sei ausfindig gemacht und getestet worden - mit negativem Ergebnis.

[Die Geschichte] [ins Rollen] brachte ein Telefonanruf beim Stadtammann. (A00/SEP.60614)

Schließlich finden sich auch Belege, bei denen keines der Vorfeldelemente die Funktion eines Aboutness-Topiks hat. Kandidaten für diese Kategorie sind die Beispiele (17), (28)(30) sowie (35) und (36).

(35) In Ludwigshafen ereigneten sich sieben Unfälle mit Blechschäden, in Worms wurden ebenfalls sieben, in Neustadt sechs und in Landau zwei Karambolagen registriert.

[Glimpflich] [davon] kamen Heidelberg und der Rhein-Neckar-Kreis. (M99/JAN.02892)

(36) Selbst wenn Brauer gentechnisch manipulierten Hopfen oder Braugerste kaufen wollten, ginge das gar nicht, denn beides stehe ,zum heutigen Zeitpunkt" nicht zur Verfügung. [...] [Ganz] [aus dem Auge] will man das Thema trotz der „ermutigenden Ergebnisse“ nicht verlieren [...] (NUN99/MAI.00856)

Vorfeldelemente wie in (35) können nur als Topik analysiert werden, wenn man einen Topikbegriff zugrunde legt, bei dem fehlende Referenzialität kein Ausschlusskriterium ist (so bezeichnet z.B. „I-Topik“ einen Ausdruck, der mit dem steigenden Akzent der Hutkontur markiert und kontrastiv interpretiert wird, ohne dass er referenziell sein muss, vgl. Jacobs (1997); ähnliches gilt für kontrastive Topiks bei Büring (1997)). Mit einer solchen Definition ließen sich die Vorfeldelemente in Beispiel (35) und (36) noch als (I-)Topik analysieren, die Beispiele (17) und (28)-(30) haben jedoch kein eindeutig kontrastives Element im Vorfeld.

Zusammenfassend lässt sich festhalten, dass typischerweise eines der Vorfeldelemente als (Aboutness-)Topik interpretiert werden kann. Zwei topikale Elemente im Vorfeld sind selten. ${ }^{11}$ Eher schon tritt der Fall ein, dass beide Elemente keine Aboutness-Topiks sind,

11 Einige Theorien lassen generell nicht mehr als ein Aboutness-Topik pro Satz zu, vgl. z.B. Götze et al. (2007). Aus einer solchen Perspektive ist der Befund erwartet. 
entweder weil sie nicht referieren oder weil eine Topikinterpretation im Kontext nicht angebracht erscheint. Topiks treten innerhalb eines mehrfachen Vorfelds tendenziell rechts auf; eine Beschränkung auf diese Position, wie von Speyer (2008) vorgeschlagen, wird jedoch den Daten nicht gerecht.

\subsection{Diskussion}

Wie verhalten sich die erhobenen Daten zu Hypothesen über mehrfache Vorfeldbesetzung, die in der Literatur vorgeschlagen wurden? Nach Engel (1970, S. 81) dienen „[s]olche Vorfeldsequenzen [...] der kontrastiven Hervorhebung. Wenn mehrere Elemente zugleich kontrastiv hervorgehoben werden sollen, kann von dieser Möglichkeit Gebrauch gemacht werden“. Diese Vermutung lässt sich mit den vorhandenen Daten nicht untermauern. Zwar finden sich Bespiele, wie in (37), auf die eine solche Beschreibung zutrifft (wenn es sich hier überhaupt um MVB handelt, nicht um Koordination), beim derzeitigen Stand der Datensammlung lässt sich jedoch die große Mehrheit der Belege nicht auf diese Weise erklären.

(37) Seine Firma Lightline halte bei Open-Air-Shows einen Sicherheitsabstand zu Flughäfen und informiere die Flugsicherung im Vorhinein. [...] Sein bester, gerade mal schuhkartongroßer Lichtshow-Laser kostet 20.000 Euro. Aber Stümper weiß:

,[Gebraucht] [im Internet] sind solche High-Power-Laser mit exzellenter Strahlenqualität schon ab 1000 Euro zu erwerben." (http://www.spiegel.de/wissenschaft/ mensch/0,1518,544807,00.html, 02/04/2008)

Ebenso problematisch ist der Vorschlag von Speyer (2008), dass nur Rahmensetzer, Topiks und kontrastive Elemente ins Vorfeld gelangen können. Dagegen sprechen Beispiele wie (18), (29) und (30), bei denen sich argumentieren lässt, dass die Vorfeldelemente keine dieser drei Funktionen haben. In diesen Fällen treten die Mehrfachbesetzungen in diskurseröffnenden Sätzen auf, was eine Interpretation als Topik zwar nicht ausschließt, jedoch in den gegebenen Kontexten unwahrscheinlich macht. Ebenso wenig fungieren die vorangestellten Elemente als Rahmensetzer, und eine kontrastive Interpretation bietet sich auch nicht an. Die Mehrfachbesetzungen in (23b) und (17) treten zwar nicht in diskursinitialen Sätzen auf, es lässt sich jedoch auch für diese Beispiele argumentieren, dass das Material im Vorfeld keine der von Speyer vorgeschlagenen Funktionen hat. Sollte es zutreffen, dass sich zumindest einige Fälle von mehrfacher Vorfeldbesetzung besser durch Eigenschaften postverbaler Konstituenten oder des gesamten Satzes erklären lassen (wie Bildhauer/Cook 2010 vorschlagen), wäre dies problematisch für merkmalsgetriebene Theorien von Bewegung, bei denen Umstellungen ausschließlich auf Eigenschaften der umgestellten Konstituenten zurückgeführt werden (z.B. Grewendorf 2002; Frey 2004a,b; Frascarelli/Hinterhölzl 2007; Speyer 2008).

\section{Andere Faktoren}

Wie oben angedeutet, ist es nicht unplausibel, anzunehmen, dass einige Fälle von mehrfacher Vorfeldbesetzung sich besser durch Eigenschaften nicht vorangestellter Konstituenten oder globaler Eigenschaften des Satzes erklären lassen, als durch Eigenschaften des Materials im Vorfeld. So könnte MVB in manchen Fällen dazu dienen, eine Topikinterpretation von Konstituenten auszuschließen, wenn eine solche als nicht angemessen für den aktuellen Stand des Diskurses empfunden wird. Ein Satz hat eine endliche Zahl möglicher Konstituentenstellungen, und mehrfache Vorfeldbesetzung könnte in den angesprochenen 
Fällen die beste Wahl aus einer Anzahl suboptimaler Linearisierungen sein. Eine solche Erklärung würde sich für die zahlreichen Sätze anbieten, in denen mehrfache Vorfeldbesetzung diskurseinleitend vorkommt, wie in Beispiel (18).

(18) [Mit seinem Lkw] [auf eine stehende Fahrzeugkolonne] fuhr Paul S. aus Absam auf.

Einige Linearisierungsvarianten:

(38a) Paul S. aus Absam fuhr [mit seinem Lkw] [auf eine stehende Fahrzeugkolonne] auf.

(38b) [Mit seinem Lkw] fuhr Paul S. aus Absam [auf eine stehende Fahrzeugkolonne] auf.

(38c) [Auf eine stehende Fahrzeugkolonne] fuhr Paul S. aus Absam [mit seinem Lkw] auf.

(38d) Paul S. aus Absam fuhr [auf eine stehende Fahrzeugkolonne] [mit seinem Lkw] auf.

(38e) [Auf eine stehende Fahrzeugkolonne] fuhr [mit seinem Lkw] Paul S. aus Absam auf.

(38f) [Mit seinem Lkw] fuhr [auf eine stehende Fahrzeugkolonne] Paul S. aus Absam auf.

Variante (38a) legt eine Topikinterpretation für das Subjekt nahe, die in diesem Kontext unpassend ist. Die Varianten (38b) und (38c) legen kontrastive Lesarten für die PPen nahe, von denen man annehmen kann, dass sie im gegebenen Kontext ebenfalls unerwünscht sind. Variante (38d) legt ebenfalls einen Topikstatus für das Subjekt nahe, zusätzlich evtl. eine kontrastive Interpretation der finalen PP. Die Varianten (38e) und (38f) sind in diesem Kontext möglicherweise Alternativen zu (18), wirken jedoch markiert. Unter diesen Umständen kann (18) die beste Wahl sein, die MVB wäre in diesem Fall einer Vermeidungsstrategie zuzuschreiben.

Ein anderer Faktor, der mehrfache Vorfeldbesetzung begünstigen könnte, ist die fehlende Möglichkeit von VP-Voranstellungen bei einfachen Verbformen.

(39) [Ganz aus den Augen verloren] haben sie sich nicht. (RHZ98/MAI.26283)

(40a) [Ganz] [aus den Augen] verlieren sie sich nicht.

(40b) [Ganz aus den Augen verlieren] tun sie sich nicht.

Variante (40b) gilt als markiert und steht in geschriebener Sprache normalerweise nicht als Option zur Verfügung, so dass nur Variante (40a) bleibt, um dem Prädikat eine informationsstrukturelle Rolle vergleichbar der in (39) zuzuweisen. Diese Erklärung reicht für sich genommen jedoch auch nicht aus, denn mehrfache Vorfeldbesetzung findet sich auch in Sätzen, in denen ein nicht-finites Verb die Voranstellung der gesamten VP ermöglichen würde. In diesem Zusammenhang liegen für einen eingeschränkten Bereich Zahlen ${ }^{12}$ vor (Tabelle 1), vor deren Hintergrund die Annahme in Frage gestellt werden muss, nach der die Voranstellung mehrerer Konstituenten mit einem nicht-finitem Verb immer den „kanonischen“ Fall darstellt, dem die mehrfache Vorfeldbesetzung als ,nicht-kanonisch“ gegenüber steht. Vergleicht man für die Konstruktion [ganz auf NP verzichten] nur die Fälle mit periphrastischen Verbformen, kommt die MVB in den Daten häufiger vor als die entsprechende Voranstellung mit nicht-finitem Verb.

\begin{tabular}{|l|l|l|l|l|l|}
\hline & ADV & PP & ADV + PP + $\mathrm{V}_{\text {inf }}$ & ADV + PP & Total \\
\hline einfach & 5 & 55 & 0 & 19 & 79 \\
\hline periphrastisch & 53 & 95 & 50 & 84 & 282 \\
\hline Total & 58 & 150 & 50 & 103 & 361 \\
\hline
\end{tabular}

Tabelle 1: Verteilung der Vorfeldbesetzungen für [ganz auf NP verzichten]

12 Alle Daten in diesem Abschnitt beziehen sich auf den öffentlich zugänglichen Teil des W-Archivs am IDS Mannheim. 
Darüber hinaus fällt auf, dass mehrfache Vorfeldbesetzungen mit einem prädikativen Element im Vorfeld relativ häufig vorkommen, vgl. (41)-(43). Ebenso ist das häufige Auftreten von Idiomen mit mehrfacher Vorfeldbesetzung beobachtet worden (vgl. St. Müller 2003), illustriert mit Belegen aus der aktuellen Datensammlung in (44)-(46). Eine mögliche Generalisierung (die genauer zu überprüfen wäre) ist, dass MVB eher auftritt, wenn sich eines der Vorfeldelemente als Teil des Prädikats analysieren lässt.

(41) Olympiasieger Nigeria, Marokko und Tunesien sind die ersten drei Mannschaften, die sich für die Fußball-WM-Endrunde 1998 in Frankreich qualifiziert haben.

[Automatisch] [Fixstarter] sind ja Titelverteidiger Brasilien und die Auswahl des Gastgeberlandes. (V97/JUN.30501)

(42) Die musikalische Begleitung besorgt Norbert Biermann am Klavier. Das Programm wird moderiert von Yvonne Hotz, deren „Lückenfüller“ vor kurzem erst das Bürstädter Publikum begeisterten. Organisiert hat die Gala der Altenheim-Leiter Günter Schwering. [...] [Auf den ersten Blick] [ein Wagnis] war das Engagement des Gospelchores. (M03/JUN.38968)

(43) Trinkfreudige Großstädter haben den deutschen Espressomarkt im vergangenen Jahr um 20 Prozent wachsen lassen.

[Bundesweit] [Spitzenreiter] sind die Süddeutschen. (NUZ08/AUG.00218)

(44) Inter Mailand mit dem Brasilianer Ronaldo und dem Chilenen Zamorano im Angriff, dahinter noch Jungstar Ventola, mit Winter, Sousa und Djorkaeff ist das Starensemble der Liga. Der Verein hielt sich mit Neuverpflichtungen insgesamt zurück, holte aber aber Italiens Liebling Robert Baggio.

[Alles] [in den Schatten] stellt Lazio Rom. (R98/SEP.73070)

(45) Weil er glaubte, es sei seine Frau, öffnete der Pensionist. Zwei dunkelhaarige Frauen drängten ihn durch den Vorraum in die Küche. Eine von ihnen wedelte mit einem Stofftaschentuch vor seinem Gesicht herum, um ihn abzulenken. Weil der 84jährige zum Gehen eine Krücke braucht, konnte er sich nicht wehren.

[Dem Spuk] [ein Ende] bereitete seine resolute Gattin. (N97/NOV.46203)

(46) Dennoch warnte Assauer davor, vom Ziel WM-Titel abzurücken: „Wenn man bei einer WM im eigenen Land nicht über das Ziel Titel reden darf, dann muss man aufhören oder Basketball spielen."

[Den Finger] [in die Wunde] legte dagegen Werder Bremens Manager Klaus Allofs (Werder Bremen). (M06/MAR.17788)

Idiome verhalten sich jedoch nicht einheitlich in Bezug auf die mehrfache Vorfeldbesetzung, so dass hier weitere Faktoren eine Rolle zu spielen scheinen, insbesondere solche, die sich auf relative Häufigkeit und Kollokation beziehen. Bei einer starken Assoziation zwischen den Vorfeldelementen ist es nicht unplausibel anzunehmen, dass Sprecher die Sequenz als Chunk reanalysieren (und ähnliches könnte auch für häufige Sequenzen in Konstruktionen zutreffen, die man nicht unbedingt als Idiome klassifizieren würde). Bei einigen Idiomen finden sich kaum Einfachvoranstellungen eines (nicht-verbalen) Idiombestandteils, beispielsweise im Fall von Öl ins Feuer gießen und das Fass zum Überlaufen bringen. Tabelle 2 zeigt die Verteilung der Vorfeldbesetzungen des Idioms Öl ins Feuer gießen (gelistet sind alle Fälle, in denen einer oder mehrere Idiombestandteile im Vorfeld stehen). Die Mehrfachbesetzung des Vorfelds (NP+PP) überwiegt bei weitem die einfache Besetzung durch die NP (die überwiegend mit periphrastischen Verbformen auftritt). Beschränkt man sich auf Fälle mit periphrastischer Verbform und betrachtet nur die Alternation zwischen den Voranstellungen mit und ohne nicht-finitem Verb, finden sich beide Konstruktionen in etwa gleich häufig. 
Tabelle 3 zeigt die entsprechenden Daten für das Idiom das Fass zum Überlaufen bringen. Auch hier gibt es sehr wenige Einfachvoranstellungen, aber obwohl es sich intuitiv bei beiden Idiomen um relativ rigide Konstellationen handelt (mit wenig Variation bei NP, PP und Verb), unterscheiden sie sich deutlich: Im Fall von das Fass zum Überlaufen bringen treten die Einfachvoranstellungen fast ausschließlich mit einfachen Verbformen auf, außerdem findet sich auch die (unerwartete) Einfachvoranstellung des zweiten Idiombestandteils. Im Gegensatz zu Öl ins Feuer gießen tritt in den Fällen mit periphrastischer Verbform die Mehrfachbesetzung des Vorfelds wesentlich seltener auf als die Voranstellung mit nicht-finitem Verb.

\begin{tabular}{|l|l|l|l|l|l|}
\hline & NP & PP & NP + PP + V & NP + PP & Total \\
\hline einfach & 2 & 0 & 0 & 80 & 82 \\
\hline periphrastisch & 5 & 1 & 21 & 18 & 45 \\
\hline Total & 7 & 1 & 21 & 98 & 127 \\
\hline
\end{tabular}

Tabelle 2: Verteilung der Vorfeldbesetzung für Öl ins Feuer gießen

\begin{tabular}{|l|l|l|l|l|l|}
\hline & $\mathrm{NP}$ & $\mathrm{PP}$ & $\mathrm{NP}+\mathrm{PP}+\mathrm{V}_{\text {inf }}$ & $\mathrm{NP}+\mathrm{PP}$ & Total \\
\hline einfach & 4 & 3 & 0 & 336 & 343 \\
\hline periphrastisch & 1 & 0 & 84 & 22 & 107 \\
\hline Total & 5 & 3 & 84 & 358 & 450 \\
\hline
\end{tabular}

Tabelle 3: Verteilung der Vorfeldbesetzung für das Fass zum Überlaufen bringen

Anhand von Idiomen der Form [NP PP V] (X ins Feuer gießen, X unter den Tisch kehren, $X$ ins Dunkel bringen) untersuchen Zeschel/Bildhauer (i.Vorb.) den Zusammenhang zwischen der Fixiertheit eines Idioms und der relativen Häufigkeit von Mehrfachvoranstellungen. Dabei wird deutlich, dass die Typfrequenz des NP-Slots (Zahl der verschiedenen NPen, die an dieser Stelle auftreten) negativ korreliert mit dem Anteil der Mehrfachvoranstellungen $(\mathrm{NP}+\mathrm{PP})$ an allen Voranstellungen $(\mathrm{NP}, \mathrm{PP}, \mathrm{NP}+\mathrm{PP}+\mathrm{V}, \mathrm{NP}+\mathrm{PP})$. Mit anderen Worten: Idiome mit relativ rigider NP+PP-Sequenz treten signifikant häufiger mit mehrfacher Vorfeldbesetzung auf (die beiden oben illustrierten Idiome befinden sich - trotz ihrer Unterschiede - beide am extremen Ende der Skala, wenn man ihr Voranstellungsverhalten mit dem strukturell ähnlicher Idiome vergleicht).

\section{Zusammenfassung}

Es wurden informationsstrukturelle Aspekte von Daten aus einer erweiterten Belegsammlung zur (scheinbar) mehrfachen Vorfeldbesetzung im Deutschen diskutiert. Die Datensammlung erlaubt es, Hypothesen aus der theoretischen Literatur nachzugehen und sie auf ihre Stichhaltigkeit zu überprüfen. Insbesondere wurde gezeigt, dass mehrfache Vorfeldbesetzung kompatibel ist mit pars-pro-toto-Voranstellung; dass (je nach Analyse) Fokus auf den gesamten Satz projizieren kann; dass die vorangestellten Konstituenten für sich genommen weder topikal, noch Rahmensetzer, noch kontrastiv sein müssen; und dass die Datensammlung von zwei Mustern dominiert wird, eine Erklärung für die MVB in diesen Fällen jedoch eher im Mittelfeld zu suchen ist. Mehrfache Vorfeldbesetzung wird als multifaktorielles Phänomen aufgefasst, und es wurden einige Faktoren vorgeschlagen, deren systematische Untersuchung weitere Aufschlüsse geben kann: MVB als Strategie zur Vermeidung unerwünschter informationsstruktureller Effekte (Topikinterpretation, Kontrast); MVB zur Erzeugung informationsstruktureller Markierungen, die auf andere Weise kaum ausgedrückt werden können (kontrastive Hervorhebung der VP in Sätzen ohne infinites 
Verb); in diesem Zusammenhang auch die Frage nach der Rolle von Kanal (geschriebene Sprache) und Textsorte (bisher wurde ausschließlich Zeitungstext untersucht); schließlich die Rolle von Kollokation (zwischen den beiden Vorfeldelementen; zwischen einem Vorfeldelement und dem Verb). Die vorliegende Bestandsaufnahme unter informationsstrukturellen Aspekten sowie die Datensammlung können als erster Anhaltspunkt für die weitere Untersuchungen dieser Einflussgrößen dienen.

\section{Literatur}

Beneš, Eduard (1971): Die Besetzung der ersten Position im deutschen Aussagesatz. In: Moser, Hugo (Hg.): Fragen der strukturellen Syntax und der kontrastiven Grammatik. Düsseldorf: Schwann. S. 160-182.

Bildhauer, Felix/Cook, Philippa (2010): German Multiple Fronting and Expected Topic-Hood. In: Müller, Stefan (Hg.): The Proceedings of the 17th International Conference on Head-Driven Phrase Structure Grammar. Stanford: CSLI Publications. S. 68-79.

Bouma, Gosse/Hendriks, Petra/Hoeksema, Jack (2007): Focus Particles inside Prepositional Phrases. A Comparison of Dutch, English and German. In: Journal of Comparative Germanic Linguistics 10, S. 1-24.

Breindl, Eva (2008): Die Brigitte nun kann der Hans nicht ausstehen. Gebundene Topiks im Deutschen. In: Deutsche Sprache 36, S. 27-49.

Büring, Daniel (1997): The Meaning of Topic and Focus. The 59th Bridge Accent. London/New York: Routledge.

Büring, Daniel/Hartmann, Katharina (2001): The Syntax and Semantics of Focus-Sensitive Particles in German. In: Natural Language and Linguistic Theory 19, 229-281.

Cook, Philippa (2010): Topic-Comment adverbs and German partial-VP topicalization involving subjects: how the contrastive reading arises. Ms., Freie Universität Berlin.

Eisenberg, Peter (2006): Grundriss der deutschen Grammatik. Der Satz. 3. Auflage. Stuttgart: Metzler.

Engel, Ulrich (1970): Regeln zur Wortstellung. Forschungsberichte des Instituts für Deutsche Sprache 5, Institut für Deutsche Sprache, Mannheim.

Fanselow, Gisbert (1993): Die Rückkehr der Basisgenerierer. In: Groninger Arbeiten zur Germanistischen Linguistik 36, S. 1-74.

Fanselow, Gisbert (2004): Cyclic Phonology-Syntax-Interaction: Movement to First Position in German. In: Ishihara, Shinichiro/Schmitz, Michaela/Schwarz, Anne (Hg.): Interdisciplinary Studies on Information Structure. Potsdam: Universitätsverlag. S. 1-42. (= Working Papers of the SFB 632, Bd. 1).

Frascarelli, Mara/Hinterhölzl, Roland (2007): Types of Topics in German and Italian. In: Schwabe, Kerstin/ Winkler, Susanne (Hg.): On Information Structure, Meaning and Form. Generalizations across Languages. Amsterdam/Philadelphia: Benjamins. S. 87-116.

Frey, Werner (2004a): The Grammar-Pragmatics Interface and the German Prefield. In: Sprache und Pragmatik 52. Lund: Germanistisches Institut der Universität Lund. S. 1-39.

Frey, Werner (2004b): A Medial Topic Position for German. In: Linguistische Berichte 198, S. 153-190.

Götze, Michael et al. (2007): Information Structure. In: Dipper, Stefanie/Götze, Michael/Skopeteas, Stavros (Hg.): Interdisciplinary Studies on Information Structure. Potsdam: Universitätsverlag. S. 147-187. (= Working Papers of the SFB 632, Bd. 7):

Grewendorf, Günther (2002): Minimalistische Syntax. Tübingen/Basel: Francke.

Hoberg, Ursula (1997): Die Linearstruktur des Satzes. In: Eroms, Hans-Werner/Stickel, Gerhard/Zifonun, Gisela (Hg.): Grammatik der deutschen Sprache. Berlin/New York: de Gruyter. S. 1495-1680. (Schriften des Instituts für Deutsche Sprache, Bd. 7.2).

Jacobs, Joachim (1986): The Syntax of Focus and Adverbials in German. In: Abraham, Werner/de Meij, Sjaak (Hg.): Topic, Focus and Configurationality. Amsterdam: Benjamins. S. 103-127.

Jacobs, Joachim (1997): I-Topikalisierung. In: Linguistische Berichte 168, S. 91-134.

Jacobs, Joachim (2001): The dimensions of topic-comment. In: Linguistics 39, S. 641-681. 
Krifka, Manfred (1995): Scope Inversion under the Rise-Fall Contour in German. Ms., Austin.

Krifka, Manfred (2007): Basic Notions of Information Structure. In: Féry, Caroline/Fanselow, Gisbert/Krifka, Manfred (Hg.): Interdisciplinary Studies on Information Structure. Potsdam: Universitätsverlag. S. 13-56. (= Working Papers of the SFB 632, Bd. 6).

Müller, Gereon (1998): Incomplete Category Fronting. A Derivational Approach to Remnant Movement in German. Dordrecht/Boston/London: Kluwer. (= Studies in Natural Language and Linguistic Theory, Bd. 42).

Müller, Gereon (2004): Verb-Second as vP-First. In: The Journal of Comparative Germanic Linguistics 7 , S. 179-234.

Müller, Stefan (2003): Mehrfache Vorfeldbesetzung. In: Deutsche Sprache 31, S. 29-62.

Müller, Stefan (2005a): Zur Analyse der scheinbar mehrfachen Vorfeldbesetzung. In: Linguistische Berichte 203, S. 297-330. (http://hpsg.fu-berlin.de/ stefan/Pub/mehr-vf-lb.html), 18. Mai 2011.

Müller, Stefan (2005b): Zur Analyse scheinbarer V3-Sätze. In: d'Avis, Franz-Josef (Hg.): Deutsche Syntax: Empirie und Theorie. Symposium Göteborg 13:-15: Mai 2004. 173-194. (= Göteborger Germanistische Forschungen, Bd. 46). (http://hpsg.fu-berlin.de/ stefan/Pub/goeteborg.html).

Nunberg, Geoffrey/Sag, Ivan A./Wasow, Thomas (1994): Idioms. In: Language 70, S. 491-538.

Pasch, Renate et al. (2003): Handbuch der deutschen Konnektoren. Linguistische Grundlagen der Beschreibung und syntaktische Merkmale der deutschen Satzverknüpfer (Konjunktionen, Satzadverbien und Partikeln). Berlin/New York: de Gruyter. (= Schriften des Instituts für Deutsche Sprache).

Reis, Marga (2005): On the Syntax of So-Called Focus Particles in German. In: Natural Language and Linguistic Theory 23, S. 459-483.

Rooth, Mats (1985): Association with focus. Dissertation, University of Massachusetts at Amherst.

Rooth, Mats (1992): A Theory of Focus Interpretation. In: Natural Language Semantics 1, S. 75-116.

Selkirk, Elisabeth (1995): Sentence Prosody. Intonation, Stress and Phrasing. In: Goldsmith, John A. (Hg.): The handbook of phonological theory. Cambridge, MA.: Blackwell. S. 550-569.

Speyer, Augustin (2008): Doppelte Vorfeldbesetzung im heutigen Deutsch und im Frühneuhochdeutschen. In: Linguistische Berichte 216, S. 455-485.

van de Velde, Marc (1978): Zur mehrfachen Vorfeldbesetzung im Deutschen. In: Conte, Maria-Elisabeth/ Ramat, Anna Giacalone/Ramat, Paolo (Hg.): Wortstellung und Bedeutung: Akten des 12. Linguistischen Kolloquiums, Pavia 1977. Tübingen: Niemeyer. S. 131-141.

Zeldes, Amir et al. (2009): ANNIS: A Search Tool for Multi-Layer Annotated Corpora. In: Proceedings of Corpus Linguistics 2009, July 20-23, Liverpool, UK. (http://ucrel.lancs.ac.uk/ publications/cl2009/358_ FullPaper.doc).

Zeschel, Arne/Bildhauer, Felix (i.Vorb.): Idiomaticity and multiple fronting in German.

Felix Bildhauer

Freie Universität Berlin

Institut für Deutsche und Niederländische Philologie

Habelschwerdter Allee 45

D-14195 Berlin

E-Mail: Felix.Bildhauer@fu-berlin.de 